\title{
Correlation of Programmed Death Ligand-1 (PD-L1) Expression with Clinicopathological Features in Non-Small Cell Lung Carcinoma: Experience from a Tertiary Cancer Care Center in India
}

\author{
Hafsa Saif ${ }^{*}$, K. S. Rajesh Kumar², Suhail Sayeed Mufti², Ritesh Giri³, V. Hrishi², Vinu Sarathy4, \\ Veena Ramaswamy5, Diganta Hazarika6, Radheshyam Naik ${ }^{2,7}$ \\ ${ }^{1}$ Department of Pathology, HealthCare Global (HCG) Hospital, Bengaluru, India \\ ${ }^{2}$ Department of Translational Medicine \& Therapeutics, Health Care Global (HCG) Hospital, Bengaluru, India \\ ${ }^{3}$ Department of Clinical Pharmacy, Health Care Global (HCG) Hospital, Bengaluru, India \\ ${ }^{4}$ Department of Medical Oncology, Health Care Global (HCG) Hospital, Bengaluru, India \\ ${ }^{5}$ Histopathology Department, Health Care Global (HCG) Hospital, Bengaluru, India \\ ${ }^{6}$ Department of Translational Medicine \& Therapeutics, Health Care Global (HCG) Hospital, Bengaluru, India \\ ${ }^{7}$ Department of Medical Oncology, Hematology and BMT, Bengaluru, India \\ Email: ^hafsasaif@gmail.com, rajesh.k@hcgel.com,drmufti.s@hcgel.com, riteshgiri1711@gmail.com,drhrishi.v@hcgel.com, \\ drveena.r@strandls.com,dhazarika@hcgel.com,radheshyam_n@yahoo.com
}

How to cite this paper: Saif, H., Kumar, K.S.R., Mufti, S.S., Giri, R., Hrishi, V., Sarathy, V., Ramaswamy, V., Hazarika, D. and Naik, R. (2020) Correlation of Programmed Death Ligand-1 (PD-L1) Expression with Clinicopathological Features in Non-Small Cell Lung Carcinoma: Experience from a Tertiary Cancer Care Center in India. Journal of Cancer Therapy, 11, 172-187.

https://doi.org/10.4236/jct.2020.113015

Received: February 24, 2020

Accepted: March 24, 2020

Published: March 27, 2020

Copyright $\odot 2020$ by author(s) and Scientific Research Publishing Inc. This work is licensed under the Creative Commons Attribution International License (CC BY 4.0).

http://creativecommons.org/licenses/by/4.0/ (c) (i) Open Access

\begin{abstract}
Background: According to the GLOBOCAN 2018 report, the estimated incidence of lung cancer in India was 67,795 in both sexes. The treatment of advanced Non-small cell lung cancer (NSCLC) saw a major paradigm shift with recent advances in molecular-targeted therapy. Immune checkpoint blockade therapy is one such novel strategy with promising clinical benefits in advanced NSCLC. Programmed cell death receptor-1/Programmed cell death ligand-1 (PD-1/PD-L1) pathway is one such checkpoint and has thus become the current area of interest in the treatment of lung carcinoma. The PD-1/ PD-L1 pathway is under active investigation as it represents a promising therapeutic target in NSCLC. The expression of PD-L1 in tumor cells has been suggested as a predictive marker of the clinical response to PD-1/ PD-L1-targeted therapy. Methods: This study was carried out at the Department of Pathology, Health Care Global Specialty Hospital, Bangalore from May 2018 to May 2019. In this study, we analyzed pattern of PD-L1 expression by immunohistochemistry testing using our own Laboratory Developed Test (LDT) in NSCLC patients. Results: Our study group comprised 50 pa-
\end{abstract}


tients of NSCLC. Among our study population, $40 \%$ of the patients exhibited PD-L1 immunopositivity $(\geq 1 \%)$ with $28 \%$ of them having any expression (TPS $1 \%-49 \%$ ) and $12 \%$ of them having a high expression (TPS $\geq 50 \%$ ) of PD-L1. Majority of them exhibited adenocarcinoma type of NSCLC under which the solid subtype showed a direct correlation with PD-L1 positivity (p-value: 0.004 ) with a poorly differentiated tumor histology being common in our population in relation to PD-L1 positivity (p-value: 0.043 ). PD-L1 expression did not correlate with age, gender, smoking status or clinical stage in our study. No association was found between tumor histology (SCC or AC) and driver mutation status with expression of PD-L1 in the present study. Conclusions: In our study, PD-L1 immunopositivity was found in $40 \%$ of patients and majority of them exhibited adenocarcinoma type of NSCLC. There was no correlation of PD-L1 expresion with age, gender, clinical stage, smoking status and tumor histology.

\section{Keywords}

Lung Cancer, PD-L1, IHC, Biomarker, Immunotherapy

\section{Introduction}

Lung cancer, especially non-small cell lung cancer (NSCLC), accounts for more than 1.5 million deaths per year and is the leading cause of cancer deaths in men and women worldwide [1]. According to the GLOBOCAN 2012 report, the estimated incidence of lung cancer in India was 70,275 in all ages and both sexes. More than $70 \%$ of patients are diagnosed with advanced disease, which may not be amenable to curative therapy [2].

Histologically, lung cancer is further divided into 3 major pathologic subtypes: adenocarcinoma, squamous cell carcinoma, and large cell carcinoma [3]. Adenocarcinoma accounts for $38.5 \%$ of all lung cancer cases, with squamous cell carcinoma accounting for $20 \%$ and large cell carcinoma accounting for $2.9 \%$. The incidence of adenocarcinoma has increased greatly in the past several decades, replacing squamous cell carcinoma as the most prevalent type of NSCLC [3] [4]. Although cigarette smoking is associated with all histologic types of lung cancer, in smokers the association is stronger for small cell lung cancer (SCLC) and for squamous cell carcinoma. Adenocarcinoma of the lung, on the other hand, is more common in never smokers ( $62 \%$ vs $18 \%$, based on 5144 cases) compared with smokers ( $19 \%$ vs $53 \%$ based on 21,853 cases) [5] [6] However, even among smokers, adenocarcinoma is becoming more common. An increased frequency of Epidermal Growth Factor Receptor (EGFR) mutations in lung adenocarcinomas of never smokers, especially in Asian cohorts has been reported recently [7]. Lung adenocarcinomas harbor EGFR mutations and Anaplastic Lymphoma Kinase (ALK) rearrangements almost exclusively and the identification of these molecular abnormalities is clinically relevant [8]. To im- 
prove the survival rate in NSCLC patients, novel treatment strategies or agents are needed. Immune checkpoint blockade therapy is one such novel and exciting strategy with promising clinical benefits in advanced NSCLC [9] [10]. The immune checkpoint mechanism has a critical role to play in suppressing the anti-tumor T-cell mediated immune response in the tumor microenvironment. Immunotherapy targeting the cellular immune checkpoints has thus become the current area of interest in lung carcinoma [11], one such checkpoint is the Programmed cell Death receptor-1/Programmed cell Death Ligand-1 (PD-1/PD-L1) pathway.

PD-L1 is a type 1 transmembrane protein $(\mathrm{B} 7-\mathrm{H} 1)$ that belongs to the $\mathrm{B} 7$ ligands family and may be expressed both on hematopoietic cells and nonhematopoietic cells, including endothelial, epithelial and tumor cells. PD-L1 is the principal membrane inhibitory ligand belonging to the $\mathrm{B} 7$ family, and the most widely studied in NSCLC [12]. Analyzing expression of PD-L1 by immunohistochemistry (IHC) staining has been the core strategy to select NSCLC patients for PD-L1 inhibitors. Overexpression of PD-L1 has been associated with higher response rates and better outcomes to several checkpoint inhibitors like Pembrolizumab (anti-PD-1), Nivolumab (anti-PD-1), Atezolizumab (anti-PD-L1), Durvalumab (anti-PD-L1) and Avelumab (anti-PD-L1) [8]. The overexpression of PD-L1 by tumor cells in NSCLC has been demonstrated in several large retrospective studies. The largest of these studies examined archived tumor tissue from 458 patients with stage I-IV NSCLC across all histologies using quantitative immunofluorescence (QIF) to detect PD-L1 expression on the tumor cell surface [15]. This study revealed that $32 \%$ of these samples expressed elevated PD-L1. Similar smaller retrospective studies in NSCLC using both QIF and immunohistochemistry (IHC) have reported rates of PD-L1 expression by tumor cells ranging from $27 \%$ to $58 \%$ [16] [17]. Smoking status has also been correlated with elevated PD-L1 expression. However, association between overall survival (OS) and PD-L1 expression remains unclear with reports of both an associated improvement and decrease in OS [18] [19] [20]. In a study conducted by Archana GV et al. in 2017, 89 cases of resected NSCLC were studied and immunopositivity for PD-L1 in tumor cells (TC) was found to be $27 \%$.

In the present study, we analyzed the immunohistochemistry (IHC) expression of PD-L1 in tumor cells (TC) of Squamous cell carcinoma (SCC), Adenocarcinoma (AC) and other NSCLC histological subtypes. This study also correlates the PD-L1 expression in association with the baseline clinical data of the patients and with different histopathological characteristics of the tumor.

\section{Materials and Methods}

This was a hospital based study approved by Institutional Ethics Committee, which was carried out at the Department of Pathology, Health Care Global Specialty Hospital, Bangalore from May 2018 to May 2019. The study group comprised of 50 patients with Non-Small Cell Lung Carcinoma presenting to HCG 
Hospital, Bangalore. Patients included in this study were at least 18 years of age, histologically confirmed for NSCLC, those who were untreated and newly diagnosed. An ideal biopsy sample in this study was defined as a minimum of 100 tumor cells which were required for the interpretation of the IHC in terms of Tumor Proportion Score (TPS) for PD-L1 expression. Hence, too tiny biopsies ( $<100$ tumor cells) were excluded from this study. Also, post-chemotherapy/ radiation cases, Fine Needle Aspiration Cytology (FNAC) samples, decalcified tissues, specimens from the metastatic sites (ex: lymph nodes) and those with Small Cell Lung Carcinoma (SCLC) or Large Cell Neuroendocrine Carcinoma (LCNEC), were not considered for this study.

A detailed history including age, gender, clinical symptoms, radiological findings, PET scan and data of other relevant investigations were collected from patient case files. For the retrospective study, formalin fixed paraffin embedded tissue blocks and/or slides (Hematoxylin and Eosin and IHC) were recovered and reviewed for the final diagnosis of Non-Small Cell Lung Carcinoma \& its subtypes. For the prospective cases, small biopsies (CT guided core biopsy) and resected (lobectomy) specimens from patients with lung carcinoma were processed and studied. These were stained with Hematoxylin \& Eosin and analyzed for subtyping of the tumor using morphology and relevant immunohistochemistry, if necessary. For small biopsies, histopathological characteristics like tumor histological type, grade of differentiation, and subtype (for adenocarcinoma cases) were determined. For resections, similar histopathological characteristics including tumor size were analyzed and tabulated. Those cases which fulfilled the inclusion criteria were subjected to immunohistochemistry testing for PD-L1.

\section{Immunohistochemistry (IHC)}

IHC was performed on 3 - 5 micron sections of formalin fixed and paraffin embedded (FFPE) tissues using Rabbit monoclonal antibody (Clone CAL10) by BIOCARE. Due to the scarcity of FDA approved testing kits in India, our own Laboratory Developed Test (LDT), tested and validated in our laboratory was used. Tonsillar tissue or PD-L1 positive lung adenocarcinoma tissue was used as positive control and was taken on the same slide as patient sample (away from marking area). Single negative control was run by omitting primary antibody and replacing diluent solution (BSA) in which the primary antibody was diluted (Figure 1).

Tissues sectioned and attached to slides, were dewaxed if paraffin embedded, treated with an antigen retrieval solution [EDTA buffer (1 Mm, $\mathrm{pH} 8)$ ] if required, blocked with a proteinaceous blocking solution (3\% BSA) and then incubated with the primary antibody. The bound primary antibody was detected by the addition of secondary antibody conjugated with horseradish peroxidise polymer and $\mathrm{DAB}$ substrate. When adequate colour developed, the slides were washed in water to stop the reaction, counterstained with Harris Haematoxylin, and covered with a mounting medium (DPX). Semi-automated method, Intellipath autostainer was used to stain the IHC. The slides were scanned using Philips Digital Ultrafast Scanner and whole slide images were generated. Digital 


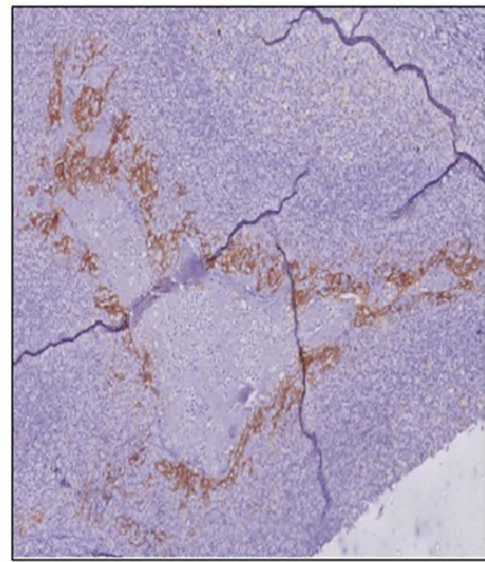

PD-L1, Positive control

(Tonsillar Crypt) (10x)

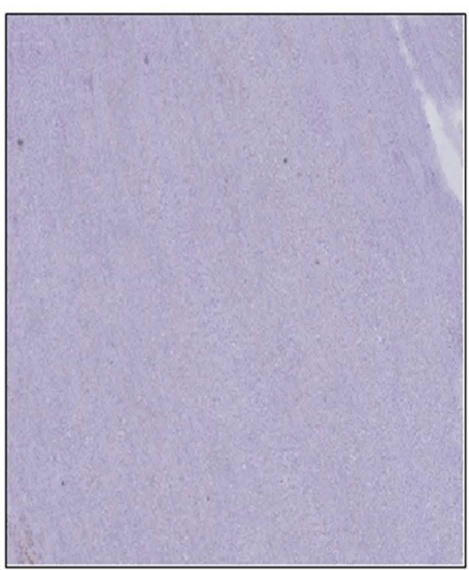

PD-L1 Negative $(<1 \%)(5 \times)$

Figure 1. PD-L1 positive control (Tonsillar tissue) and PD-L1 negative control.

images of the selected image histology were then photographed using the snapshot tool in the image management system software.

\section{Immunohistochemical Staining Analysis}

PD-L1 immunopositivity was determined and interpreted using the Tumor Proportion Score (TPS) of $<1 \%$ (no expression), 1\% - 49\% (any expression) and $>50 \%$ (high expression) (Figure 2). Any membrane (complete circumferential or partial linear plasma membrane) or cytoplasmic staining, at any intensity was considered positive for PD-L1.

\section{Statistical Analysis}

Descriptive statistics were used to describe data such as patient demographics and tumor-specific variables. Continuous variables were expressed as Mean and Standard deviation and categorical variables were expressed as Frequency and Percentage.

Statistical analysis was done to assess correlation of PDL-1 expression with clinicopathological and histopathological features. In these inferential statistics, Chi-square test/Fisher's Exact test was used to associate between the categorical variables. All statistical analyses were tested at $95 \%$ confidence interval and a P-value of $<0.05$ was considered as statistically significant. SPSS Software, Version 23 (2015) was used for the analysis.

\section{Results}

\section{Patient Characteristics}

A total of 50 patients were included in our study and all of the patients' data in this study were analyzed for various demographic, clinicopathological and histopathological characteristics as mentioned in Table 1.

Of the 50 patients included in the study, 11 patients (22\%) were aged below 50 years, 29 patients (58\%) were between 50 - 70 years, and 10 patients (20\%) were aged above 70 years. The mean age for lung carcinoma was found to be $60.92 \pm$ 13.46 years, with the youngest patient being 21 years old and the oldest being 93 
Table 1. Patient \& tumor characteristics.

\begin{tabular}{|c|c|c|c|}
\hline Characteristics & $\begin{array}{l}\text { Number }(\mathrm{N}) \\
{[\text { Total }=50]}\end{array}$ & $\begin{array}{c}\text { Percentage } \\
\text { (\%) }\end{array}$ & $\begin{array}{c}\text { Mean } \pm \text { SD } \\
(\text { Min - Max) }\end{array}$ \\
\hline \multicolumn{4}{|c|}{ Age } \\
\hline$<50$ Years & 11 & 22.0 & \multirow{3}{*}{$\begin{array}{c}60.92 \pm 13.46 \\
(21-93)\end{array}$} \\
\hline $50-70$ Years & 29 & 58.0 & \\
\hline \multirow[t]{2}{*}{$>70$ Years } & 10 & 20.0 & \\
\hline & \multicolumn{3}{|l|}{ Gender } \\
\hline Male & 31 & 62.0 & \multirow{2}{*}{ NA } \\
\hline \multirow[t]{2}{*}{ Female } & 19 & 38.0 & \\
\hline & \multicolumn{3}{|c|}{ Smoking History } \\
\hline Yes & 13 & 26.0 & \multirow{2}{*}{ NA } \\
\hline No & 37 & 74.0 & \\
\hline \multicolumn{4}{|c|}{ Pack Years } \\
\hline$<20$ Years & 2 & 15.4 & \multirow{3}{*}{ NA } \\
\hline $20-40$ Years & 7 & 53.8 & \\
\hline$>40$ Years & 4 & 30.8 & \\
\hline \multicolumn{4}{|c|}{ Tumor Size } \\
\hline$<3 \mathrm{~cm}$ & 7 & 14.0 & \multirow{3}{*}{ NA } \\
\hline $3-7 \mathrm{~cm}$ & 41 & 82.0 & \\
\hline$>7 \mathrm{~cm}$ & 2 & 4.0 & \\
\hline \multicolumn{4}{|c|}{ Metastasis } \\
\hline Yes & 45 & 90.0 & \multirow{2}{*}{ NA } \\
\hline No & 5 & 10.0 & \\
\hline \multicolumn{4}{|c|}{ Clinical Stage } \\
\hline Stage I & 2 & 4.0 & \multirow{4}{*}{ NA } \\
\hline Stage II & 3 & 6.0 & \\
\hline Stage III & 7 & 14.0 & \\
\hline Stage IV & 38 & 76.0 & \\
\hline \multicolumn{4}{|c|}{ Histological Type } \\
\hline Squamous Cell Carcinoma (SCC) & 7 & 14.0 & \multirow{2}{*}{ NA } \\
\hline Adenocarcinoma (AC) & 43 & 86.0 & \\
\hline \multicolumn{4}{|c|}{ Adenocarcinoma Subtypes } \\
\hline Acinar & 19 & 44.18 & \multirow{5}{*}{ NA } \\
\hline Lepidic & 1 & 2.32 & \\
\hline Papillary & 4 & 9.30 & \\
\hline Mucinous & 1 & 2.32 & \\
\hline Solid & 18 & 41.86 & \\
\hline \multicolumn{4}{|c|}{ Tumor Differentiation Grade } \\
\hline Well (G1) & 6 & 12.0 & \multirow{3}{*}{ NA } \\
\hline Moderate (G2) & 21 & 42.0 & \\
\hline Poor (G3) & 23 & 46.0 & \\
\hline \multicolumn{4}{|c|}{ Sampling Method Used } \\
\hline Core Biopsies & 48 & 96.0 & \multirow{2}{*}{ NA } \\
\hline Resections & 2 & 4.0 & \\
\hline
\end{tabular}




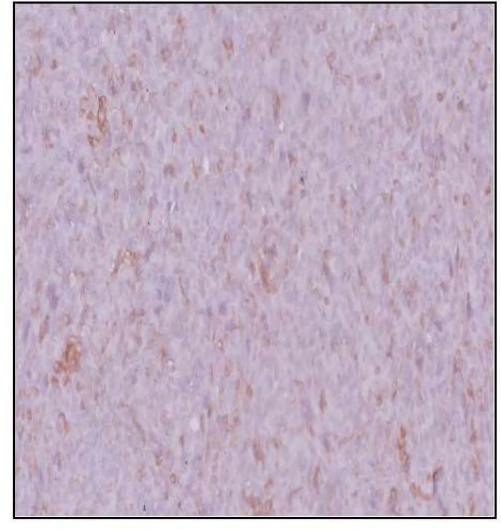

(a)

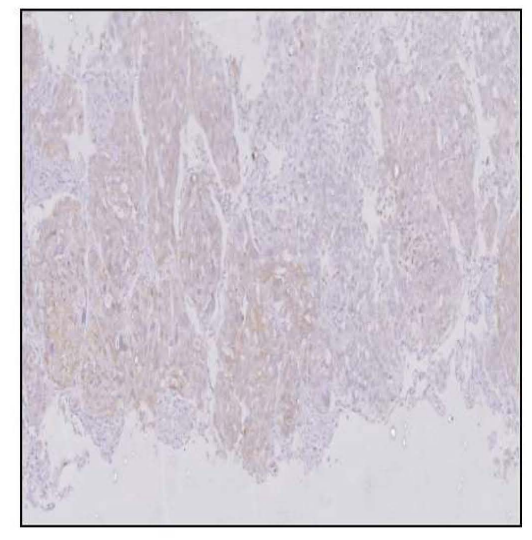

(b)

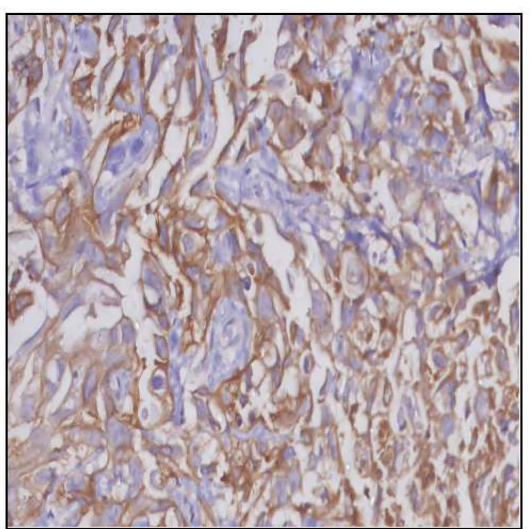

(c)

Figure 2. PD-L1 positive expression (a) 20\%, any expression (10×); (b) 30\%, any expression (10×); (c) 50\%, high expression (40×).

years of age. Out of these patients, $62 \%$ were males $(n=31)$ and $38 \%$ were females $(n=19)$, with a male to female ratio of 1.6:1. The total number of smokers in this study was $13(26 \%)$ and 37 patients (74\%) were non-smokers. Among smokers, all were males (no female smokers) and among these, 9 patients were ex-smokers and the other 4 were current/active smokers. 15.4\% $(\mathrm{n}=2)$ of the patients had a smoking history of $<20$ pack years, $53.8 \%(\mathrm{n}=7)$ had a history of 20 - 40 pack years of smoking, and $30.8 \%(n=4)$ had smoked for $>40$ pack years in their lifetime.

\section{Histopathological Features of the Tumor among Our Study Group}

Among the 50 cases, $12 \%(n=6)$ had well differentiated tumors and $42 \%(\mathrm{n}=$ 21) had moderately differentiated tumors. Majority of the cases $(46 \%, n=23)$ exhibited a poorly differentiated histology. Maximum number of patients $(76 \%$, $\mathrm{n}=38)$ belonged to clinical Stage IV. $14 \%$ of the patients $(\mathrm{n}=7)$ belonged to Stage III and a minority of patients, i.e. $6 \%(\mathrm{n}=3)$ and $4 \%(\mathrm{n}=2)$ had Stage II and Stage I disease, respectively. Majority (90\%) of the patients in this study presented with metastatic tumor $(n=45)$, with only $10 \%(n=5)$ of the patients having a tumor confined to the lung. The various sites of metastasis were lymph nodes, axial skeleton, liver, adrenal gland and colon in decreasing order of fre- 
quency. There were 43 cases of adenocarcinoma (86\%), while only 7 patients (14\%) had squamous cell carcinoma. Adenocarcinoma was the most common histological type of NSCLC in our cohort. Among the adenocarcinoma cases, acinar predominant subtype was more common $(n=19,44.18 \%)$, closely followed by solid predominant subtype $(\mathrm{n}=18,41.86 \%)$. There were 4 cases of papillary predominant adenocarcinoma $(9.3 \%)$, while lepidic and mucinous adenocarcinoma each constituted only $2.32 \%(n=1)$ of the cases, respectively. Representative $\mathrm{H} \& \mathrm{E}$ images of squamous cell carcinoma and adenocarcinoma subtypes are depicted in Figure 3.

\section{Frequency of EGFR, ALK and ROS Mutations}

Data collected on EGFR mutation, ALK rearrangements and ROS1 mutations are summarized in Table 2. Among our cases, $18 \%$ tested positive for EGFR mutation and $64 \%$ were tested negative for EGFR mutation. For ALK, data was available for 32 patients among whom 2 patients (6\%) demonstrated ALK rearrangement. Data was not available for 18 (36\%) of the cases. Whereas, mutations in ROS1 gene were tested in $46 \%$ of the patients $(n=23)$ with all cases being negative, while no data on ROS1 gene was available in $54 \%$ of the patients $(\mathrm{n}=$ 27).

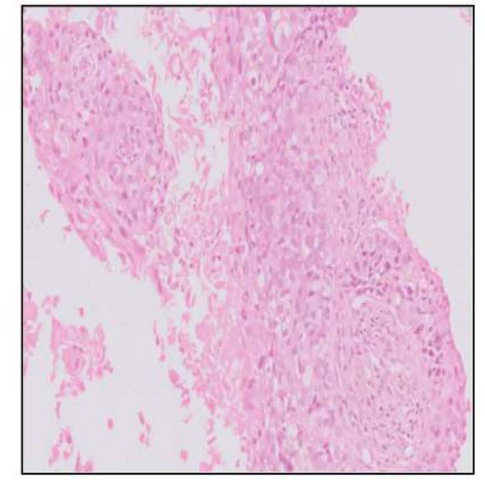

Squamous cell carcinoma moderately differentiated ( $\mathrm{H} \& \mathrm{E}, 10 \times$ )

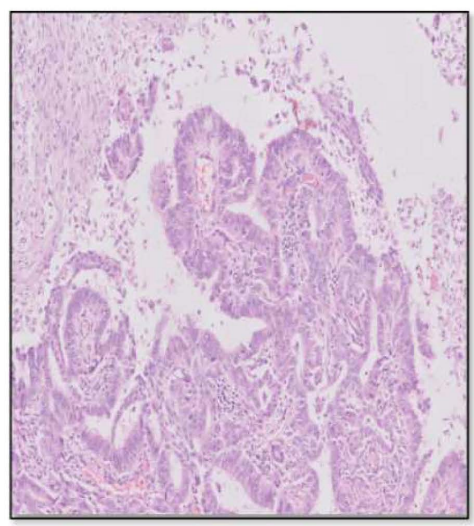

Adenocarcinoma,

Papillary subtype (H \& E, 20×)

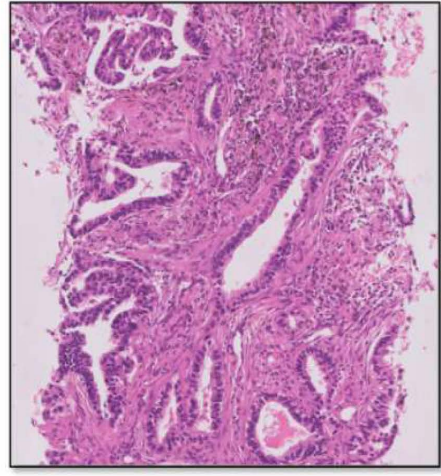

Adenocarcinoma, Acinar subtype (H \& E, 20×)

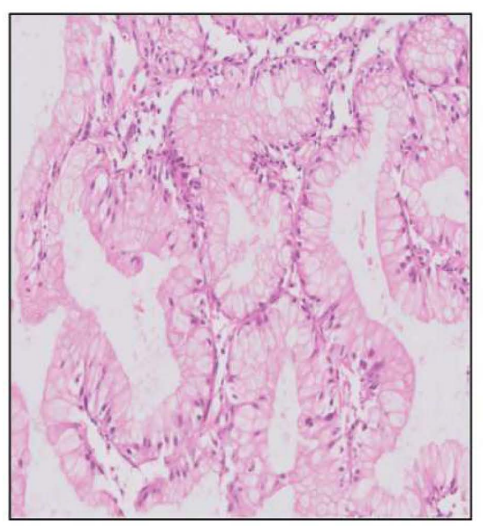

Adenocarcinoma,

Lepidic subtype (H \& E, 40×)

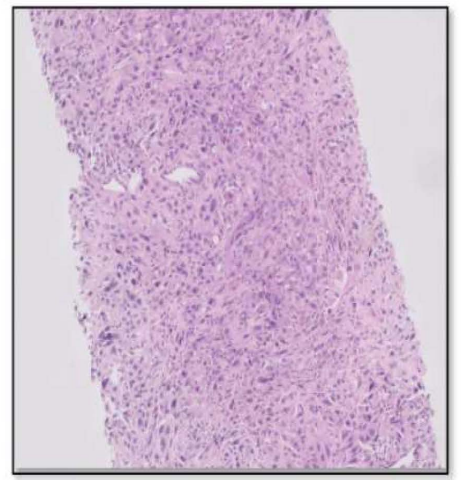

Adenocarcinoma, Solid subtype, Poorly differentiated (H \& E, 10×)

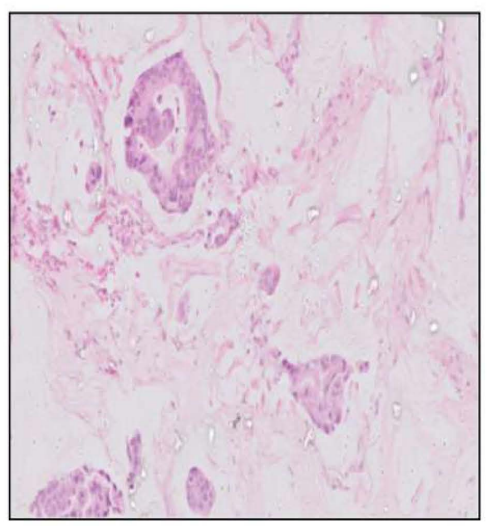

Adenocarcinoma,

Mucinous subtype (H \& E, 20×)

Figure 3. Representative H \& E images of Squamous cell carcinoma and Adenocarcinoma subtypes. 
Table 2. Frequency of EGFR, ALK and ROS1 mutations among the study group.

\begin{tabular}{ccc}
\hline Driver Mutation & Number $(\mathrm{N})$ & Percentage (\%) \\
\hline Exon 19 Deletion & EGFR Mutation & 12.0 \\
Exon 21 Mutation & 6 & 6.0 \\
Negative & 32 & 64.0 \\
Data Not Available & 9 & 18.0 \\
& ALK Rearrangement & \\
Positive & 2 & 4.0 \\
Negative & 30 & 60.0 \\
Data Not Available & 18 & 36.0 \\
& ROS1 Mutation & \\
Positive & 0 & 0.0 \\
Negative & 23 & 46.0 \\
Data Not Available & 27 & 54.0 \\
\hline
\end{tabular}

\section{PD-L1 Expression Profile}

PD-L1 positivity in our cohort was $40 \%(\mathrm{n}=20 / 50)$ with $30 \%$ of the PD-L1 positive patients showing $>50 \%$ or high PD-L1 expression (6/20), while the remaining $70 \%$ of PD-L1 positive patients showing an expression between $1 \%$ and $49 \%(14 / 20)$. Overall, $28 \%$ of the patients (14/50) showed PD-L1 expression of $1 \%-49 \%$, while $12 \%$ of the patients $(6 / 50)$ showed a high or $>50 \%$ PD-L1 expression (Table 3).

\section{Association of PD-L1 Expression with Age, Gender and Smoking Status}

In our study, the mean age of patients with PD-L1 positivity was 59.15 years while PD-L1 negative patients had a mean age of 62.10. In the group of patients $<50$ years of age, 6 cases (54.5\%) tested PD-L1 positive, while 9 patients $(31.0 \%)$ in the age group of $50-70$ years showed PD-L1 positivity. 50\% of the patients $(n=5)$ in the age group of $>70$ years showed positive PD-L1 expression. However, the association between age and PD-L1 was not found to be statistically significant (p-value 0.308). The overall positivity of PD-L1 among females was $26.3 \%(5 / 19)$ and $48.4 \%$ (15/31) among males. No significant statistical association between gender and PD-L1 expression was found in our study (p-value 0.122). Our study did not show any significant association of age and sex with PD-L1 expression. The total number of smokers in this study was 13 (26\%), all of which were males, and 37 (74\%) patients were non-smokers. All the women in the present study were non-smokers. $46.2 \%$ of smokers (6/13) were PD-L1 positive while 53.8\% (7/13) were PD-L1 negative. The association between smoking status and PD-L1 expression was not statistically significant in our study (p-value 0.236) (Table 4).

PD-L1 Expression across Various Tumor Sizes and Clinical Stages 
Table 3. PD-L1 expression among the study population.

\begin{tabular}{ccc}
\hline PD-L1 Expression & Number (n) & Percentage (\%) \\
\hline Positive & 20 & 40.0 \\
Negative & 30 & 60.0 \\
& Tumor Proportion Score (TPS) & \\
$<1 \%$ & 30 & 60.0 \\
$1 \%-49 \%$ & 14 & 28.0 \\
$>50 \%$ & 6 & 12.0 \\
\hline
\end{tabular}

Table 4. Association between PD-L1 expression and different patient characteristics.

\begin{tabular}{|c|c|c|c|c|c|c|}
\hline & \multirow{2}{*}{\multicolumn{2}{|c|}{ Patient Characteristics }} & \multicolumn{2}{|c|}{ PD-L1 } & \multirow{2}{*}{ Total } & \multirow{2}{*}{ p-Value } \\
\hline & & & Negative & Positive & & \\
\hline \multirow{6}{*}{ Age } & \multirow{2}{*}{$<50$ years } & Count & 5 & 6 & 11 & \multirow{6}{*}{$\begin{array}{l}0.308 \\
(\mathrm{NS})\end{array}$} \\
\hline & & $\%$ within $<50 \mathrm{yrs}$ & $45.5 \%$ & $54.5 \%$ & $100.0 \%$ & \\
\hline & & Count & 20 & 9 & 29 & \\
\hline & $30-70$ years & $\%$ within $50-70$ yrs & $69.0 \%$ & $31.0 \%$ & $100.0 \%$ & \\
\hline & \multirow{2}{*}{$>70$ years } & Count & 5 & 5 & 10 & \\
\hline & & $\%$ within $>70$ yrs & $50.0 \%$ & $50.0 \%$ & $100.0 \%$ & \\
\hline \multirow{4}{*}{ Gender } & \multirow{2}{*}{ FEMALE } & Count & 14 & 5 & 19 & \multirow{4}{*}{$\begin{array}{l}0.122 \\
(\mathrm{NS})\end{array}$} \\
\hline & & $\%$ within females & $73.7 \%$ & $26.3 \%$ & $100.0 \%$ & \\
\hline & \multirow{2}{*}{ MALE } & Count & 16 & 15 & 31 & \\
\hline & & $\%$ within males & $51.6 \%$ & $48.4 \%$ & $100.0 \%$ & \\
\hline \multirow{4}{*}{$\begin{array}{l}\text { Smoking } \\
\text { Status }\end{array}$} & \multirow{2}{*}{ Yes } & Count & 7 & 6 & 13 & \multirow{4}{*}{$\begin{array}{l}0.236 \\
(\mathrm{NS})\end{array}$} \\
\hline & & $\%$ within smokers & $53.8 \%$ & $46.2 \%$ & $100.0 \%$ & \\
\hline & \multirow{2}{*}{ No } & Count & 23 & 14 & 37 & \\
\hline & & $\%$ within non-smokers & $37.9 \%$ & $62.1 \%$ & $100.0 \%$ & \\
\hline \multirow{6}{*}{$\begin{array}{c}\text { Tumor } \\
\text { Size }\end{array}$} & \multirow{2}{*}{$<3 \mathrm{~cm}$} & Count & 5 & 2 & 7 & \multirow{6}{*}{$\begin{array}{l}0.778 \\
(\mathrm{NS})\end{array}$} \\
\hline & & $\%$ within $<3 \mathrm{~cm}$ & $71.4 \%$ & $28.6 \%$ & $100.0 \%$ & \\
\hline & \multirow{2}{*}{$3-7 \mathrm{~cm}$} & Count & 24 & 17 & 41 & \\
\hline & & $\%$ within $3-7 \mathrm{~cm}$ & $58.5 \%$ & $41.5 \%$ & $100.0 \%$ & \\
\hline & \multirow{2}{*}{$>7 \mathrm{~cm}$} & Count & 1 & 1 & 2 & \\
\hline & & $\%$ within $>7 \mathrm{~cm}$ & $50.0 \%$ & $50.0 \%$ & $100.0 \%$ & \\
\hline \multirow{11}{*}{$\begin{array}{c}\text { Clinical } \\
\text { Stage }\end{array}$} & \multirow{2}{*}{ STAGE I } & Count & 1 & 1 & 2 & \multirow{11}{*}{$\begin{array}{l}0.764 \\
(\mathrm{NS})\end{array}$} \\
\hline & & $\%$ within Stage I & $50.0 \%$ & $50.0 \%$ & $100.0 \%$ & \\
\hline & \multirow{2}{*}{ STAGE II } & Count & 1 & 2 & 3 & \\
\hline & & \% within Stage II & $33.3 \%$ & $66.7 \%$ & $100.0 \%$ & \\
\hline & \multirow{2}{*}{ STAGE III } & Count & 4 & 3 & 7 & \\
\hline & & $\%$ within Stage III & $57.1 \%$ & $42.9 \%$ & $100.0 \%$ & \\
\hline & \multirow{3}{*}{ STAGE IV } & Count & 24 & 14 & 38 & \\
\hline & & $\%$ within Stage IV & $63.2 \%$ & $36.8 \%$ & $100.0 \%$ & \\
\hline & & \% within G3 & 43.5 & 56.5 & $100.0 \%$ & \\
\hline & \multirow{2}{*}{ G1, G2 } & Count & 20 & 7 & 27 & \\
\hline & & $\%$ within $\mathrm{G} 1$ and G2 & 74.1 & 25.9 & $100.0 \%$ & \\
\hline
\end{tabular}


$28.6 \%$ of patients who had a tumor $<3 \mathrm{~cm}$ were PD-L1 positive $(\mathrm{n}=2)$, whereas $71.4 \%$ of these patients were PD-L1 negative $(n=5)$. Among patients whose tumor size varied between $3-7 \mathrm{~cm}, 41.5 \%(\mathrm{n}=17)$ showed PD-L1 expression while $58.5 \%(\mathrm{n}=24)$ showed no expression of PD-L1. $50 \%$ of patients with a tumor $>7 \mathrm{~cm}$ in size tested positive for PD-L1 $(\mathrm{n}=1)$ while the remaining 50\% were negative $(\mathrm{n}=1)$. Tumor size and PD-L1 expression showed no significant association (p-value 0.778) (Table 4).

Majority of the patients $(n=38)$ belonged to Stage IV in the present study. Among these Stage IV patients, 36.8\% $(\mathrm{n}=14)$ showed PD-L1 expression, while $63.2 \%(n=24)$ showed no expression of PD-L1. Out of 7 patients who belonged to clinical Stage III, $42.9 \%(n=3)$ were PD-L1 positive while $57.1 \%(n=4)$ were negative. Among Stage II patients, $66.7 \%(n=2)$ and $33.3 \%$ patients $(n=1)$ were PD-L1 positive and negative, respectively. $50 \%$ of Stage I patients $(n=1)$ showed PD-L1 expression. The association of PD-L1 with clinical stage was not significant statistically (p-value 0.764) (Table 4).

\section{Association of PD-L1 with Tumor Histology}

Out of 20 patients with PD-L1 positive expression, 17 patients (85\%) had adenocarcinoma while 3 patients (15\%) had squamous cell carcinoma histology. $60.5 \%(\mathrm{n}=26)$ of adenocarcinoma patients were PD-L1 negative and 57.1\% $(\mathrm{n}=$ 4) of squamous cell carcinoma patients were PD-L1 negative. However, no significant association was found between tumor histologic type and PD-L1 expression (p-value 0.868) (Figure 4).

Correlation of PD-L1 with Adenocarcinoma Subtype and Tumor Differentiation

Among the adenocarcinoma group of patients $(n=43), 18$ patients had solid predominant subtype. Among these 18, 12 patients showed a positive PD-L1 expression $(66.6 \%)$ and the remaining 6 were PD-L1 negative $(33.4 \%)$. The remaining subtypes of adenocarcinoma (acinar, papillary) $(n=25)$ showed PD-L1 positivity in only 5 cases (20\%) while the rest were PD-L1 negative $(80 \%, n=20)$ (Figure 5). There was a highly significant statistical association between adenocarcinoma subtype and PD-L1 expression, with the solid subtype correlating with positive PD-L1 expression (p-value 0.004) (Table 5).

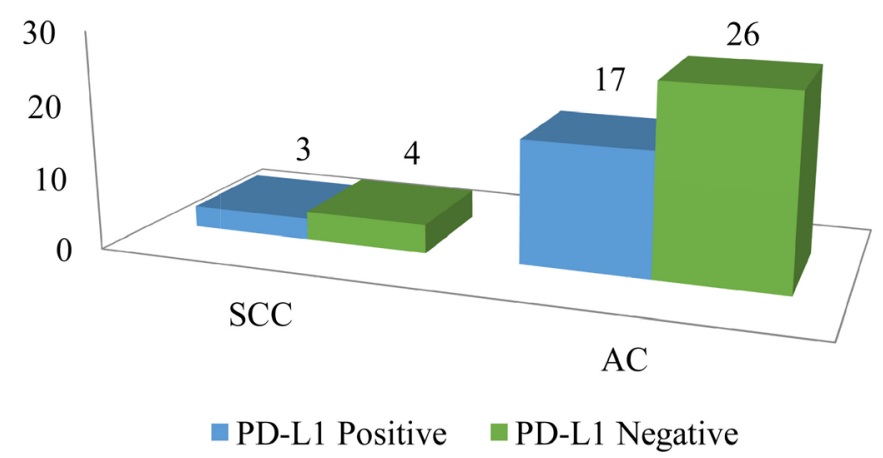

Figure 4. Association between PD-L1 expression with histologic type; Squamous Cell Carcinoma (SCC) and Adenocarcinoma (AC). 


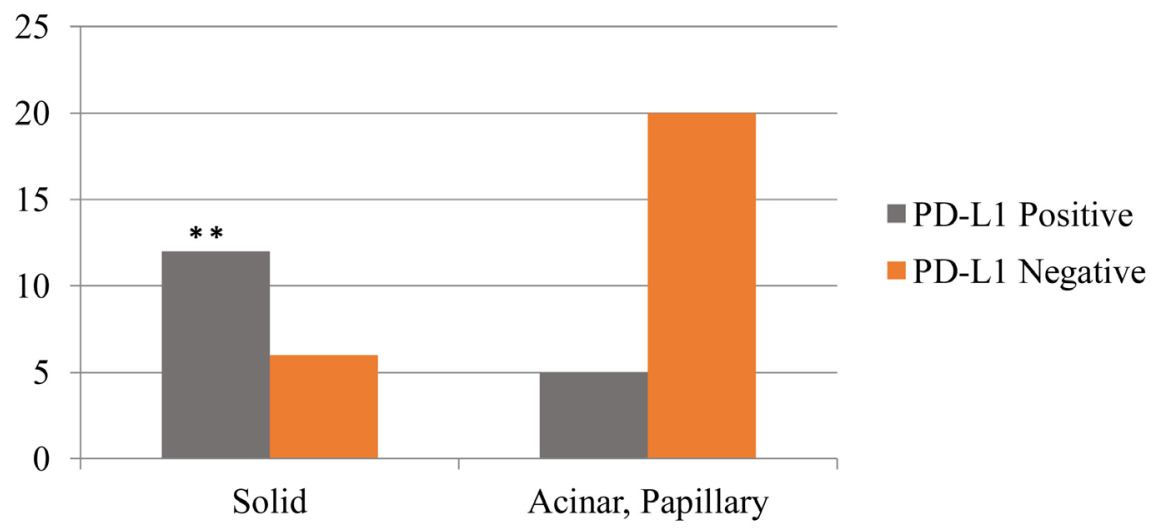

Figure 5. PD-L1 expression among histological subtypes of Adenocarcinoma.

Table 5. Association between PD-L1 expression with Adenocarcinoma subtype and Tumor Differentiation.

\begin{tabular}{|c|c|c|c|c|c|c|}
\hline \multirow{2}{*}{\multicolumn{3}{|c|}{ Tumor Characteristics }} & \multicolumn{2}{|c|}{ PD-L1 } & \multirow{2}{*}{ Total } & \multirow{3}{*}{$\mathrm{p}$-Value } \\
\hline & & & Negative & Positive & & \\
\hline \multirow{3}{*}{$\begin{array}{c}\text { Adenocarcinoma } \\
\text { Subtype }\end{array}$} & & Count & 6 & 12 & 18 & \\
\hline & Solid & $\begin{array}{c}\text { \% within } \\
\text { Solid Subtype }\end{array}$ & $33.4 \%$ & $66.6 \%$ & $100.0 \%$ & \multirow[b]{2}{*}{$0.004^{*}$} \\
\hline & $\begin{array}{l}\text { Acinar, } \\
\text { Papillary }\end{array}$ & $\begin{array}{c}\text { Count } \\
\text { \% within } \\
\text { Other Subtypes }\end{array}$ & $80 \%$ & $20 \%$ & $100.0 \%$ & \\
\hline \multirow[b]{2}{*}{$\begin{array}{c}\text { Tumor } \\
\text { Differentiation }\end{array}$} & G3 & $\begin{array}{c}\text { Count } \\
\text { \% within G3 }\end{array}$ & $\begin{array}{c}10 \\
43.5\end{array}$ & $\begin{array}{c}13 \\
56.5\end{array}$ & $\begin{array}{c}23 \\
100.0 \%\end{array}$ & \multirow[b]{2}{*}{$0.043^{*}$} \\
\hline & $\mathrm{G} 1, \mathrm{G} 2$ & $\begin{array}{l}\text { Count } \\
\text { \% within } \\
\text { G1 and G2 }\end{array}$ & 74.1 & 25.9 & $100.0 \%$ & \\
\hline
\end{tabular}

Among 27 tumors belonging to well (G1) and moderately differentiated (G2) categories, $25.9 \%$ showed PD-L1 expression $(\mathrm{n}=7)$ and $74.1 \%$ showed no expression of PD-L1 $(n=20)$. However, $56.5 \%$ of the tumors $(n=13)$ having poorly differentiated histology (G3) were positive for PD-L1 while $43.5 \%(\mathrm{n}=$ 10) had $<1 \%$ PD-L1 expression (Figure 6). This correlation was found to be statistically significant (p-value 0.043) (Table 5).

\section{PD-L1 and its Correlation with Driver Mutations}

PD-L1 expression was $>1 \%$ in 3 cases (50\%) out of 6 cases with Exon 19 mutations. Out of 3 cases with Exon 21 mutation, 1 case (33.3\%) showed PD-L1 expression while the remaining $2(66.7 \%)$ showed no expression. Among the 32 cases that tested negative for EGFR mutations, $43.8 \%(\mathrm{n}=14)$ showed positivity for PD-L1 while $56.2 \%(\mathrm{n}=18)$ were negative. There was no statistically significant association between EGFR mutations and PD-L1 expression (p-value 0.642) (Table 6). 


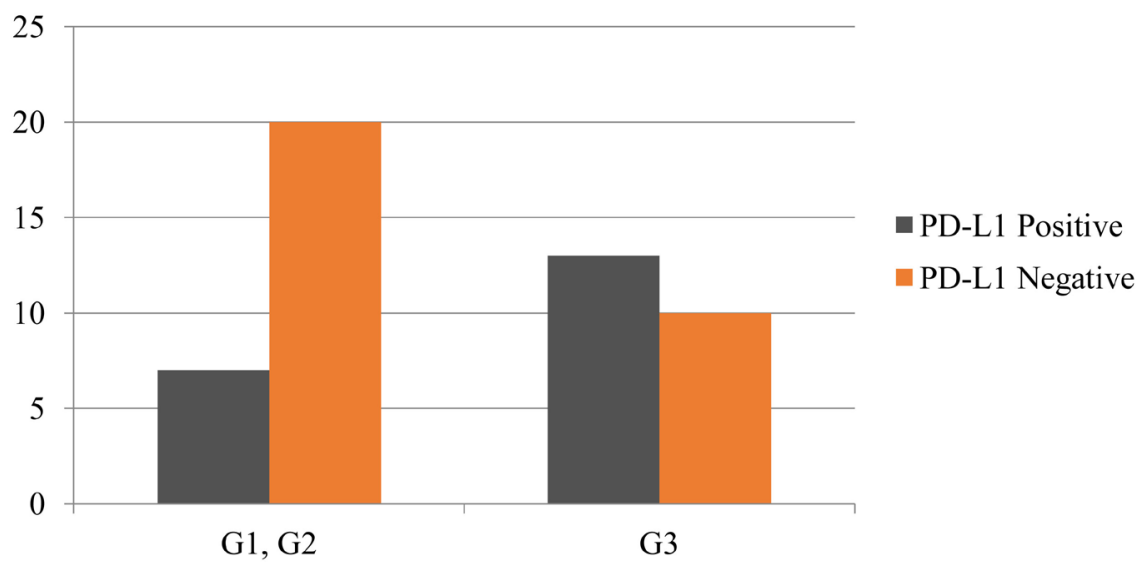

Figure 6. Association between PD-L1 and tumor differentiation.

Table 6. Association between PD-L1 expression and driver mutations.

\begin{tabular}{|c|c|c|c|c|c|c|}
\hline \multirow{2}{*}{\multicolumn{3}{|c|}{ Driver Mutation }} & \multicolumn{2}{|c|}{ PD-L1 } & \multirow{2}{*}{ Total } & \multirow{2}{*}{ p-Value } \\
\hline & & & Negative & Positive & & \\
\hline \multirow{6}{*}{$\begin{array}{c}\text { EGFR } \\
\text { mutation }\end{array}$} & Exon & Count & 3 & 3 & 6 & \multirow{8}{*}{$\begin{array}{l}0.642 \\
(\mathrm{NS})\end{array}$} \\
\hline & 19 deletion & $\%$ & $50.0 \%$ & $50.0 \%$ & $100.0 \%$ & \\
\hline & Exon & Count & 2 & 1 & 3 & \\
\hline & 21 mutation & $\%$ & $66.7 \%$ & $33.3 \%$ & $100.0 \%$ & \\
\hline & \multirow{2}{*}{ No mutation } & Count & 18 & 14 & 32 & \\
\hline & & $\%$ & $56.2 \%$ & $43.8 \%$ & $100.0 \%$ & \\
\hline \multirow{8}{*}{$\begin{array}{c}\text { ALK } \\
\text { Rearrangement }\end{array}$} & Data & Count & 7 & 2 & 9 & \\
\hline & not available & $\%$ & $77.8 \%$ & $22.2 \%$ & $100.0 \%$ & \\
\hline & No & Count & 16 & 14 & 30 & \multirow{6}{*}{$\begin{array}{l}0.415 \\
(\mathrm{NS})\end{array}$} \\
\hline & rearrangment & $\%$ & $53.3 \%$ & $46.7 \%$ & $100.0 \%$ & \\
\hline & Data not & Count & 13 & 5 & 18 & \\
\hline & available & $\%$ & $72.2 \%$ & $27.8 \%$ & $100.0 \%$ & \\
\hline & \multirow{2}{*}{ Positive } & Count & 1 & 1 & 2 & \\
\hline & & $\%$ & $50.0 \%$ & $50.0 \%$ & $100.0 \%$ & \\
\hline \multirow{6}{*}{ ROS1 Mutation } & \multirow{2}{*}{ Positive } & Count & 0 & 0 & 0 & \multirow{6}{*}{0.487 (NS) } \\
\hline & & $\%$ & $0.0 \%$ & $0.0 \%$ & $0.0 \%$ & \\
\hline & \multirow{2}{*}{ Negative } & Count & 15 & 8 & 23 & \\
\hline & & $\%$ & $65.2 \%$ & $34.8 \%$ & $100.0 \%$ & \\
\hline & \multirow{2}{*}{$\begin{array}{l}\text { Data not } \\
\text { available }\end{array}$} & Count & 15 & 12 & 27 & \\
\hline & & $\%$ & $55.6 \%$ & $44.4 \%$ & $100.0 \%$ & \\
\hline
\end{tabular}

$50 \%$ of the patients $(\mathrm{n}=1)$ having an ALK rearrangement showed PD-L1 expression, while $46.7 \%(n=14)$ of those who did not have any ALK mutation were PD-L1 positive. No statistical association was found between PD-L1 expression and ALK mutation (p-value 0.415). None of the cases had ROS1 muta- 
tion and $46.7 \%(n=14)$ of these had positive PD-L1 expression. Data was unavailable in 18 cases. The association between ROS1 and PD-L1 expression was not statistically significant (p-value 0.487 ) (Table 6).

\section{Discussion}

Our study was carried out to analyze the pattern of PD-L1 expression in NSCLC patients, in a tertiary care hospital in Bangalore. To our knowledge, there are limited studies from India which have evaluated the expression profile of this important biomarker. A meta-analysis conducted by Minghui $\mathrm{Z}$ et al. included 47 different studies on PD-L1 expression and found PD-L1 positivity rates ranging from $14.3 \%$ to $75 \%$ among NSCLC patients [2]. Our study showed an overall PD-L1 positivity of $40 \%$, which falls within this range. In a study by Archana GV et al., no correlation was found between smoking and PD-L1 expression [11]. Hence, our study showed similar results as the other Indian study. No significant association was found between tumor size and PD-L1 expression among the subjects in the present study. This finding was contrary to that demonstrated by Yunjian $\mathrm{P}$ et al. who found that there was a significant association between positive PD-L1 expression (including TPS $\geq 50 \%$ and TPS $<50 \%$ ) in the ADC cohort with large tumor size [21]. Adenocarcinoma was the most common histological type of NSCLC in the current study. PD-L1 expression, however, showed no significant correlation with histologic type. Archana GV et al. found that higher PD-L1 positivity rates were shown by sarcomatoid carcinoma and solid predominant adenocarcinomas [11] Our study is in agreement with these observations as we found a highly significant statistical association between positive PD-L1 expression (i.e. TPS $1 \%-49 \%$ and $>50 \%$ ) with solid subtype of adenocarcinoma ( $\mathrm{p}$-value 0.004 ). Thus, we can conclude that patients with solid subtype of adenocarcinoma might show a positive PD-L1 expression and are likely to benefit from immune checkpoint/anti-PD-L1 therapy. Our study demonstrated that less tumor differentiation/poorly differentiated histology (G3) was significantly associated with PD-L1 positivity ( $\mathrm{p}$-value 0.043 ). This finding is in concordance with the finding by Kentaro Inamura et al., who observed that PD-L1 expression was associated with less tumor differentiation [1]. In our study, we did not find any significant association between EGFR, ALK or ROS1 mutation status and PD-L1 positivity in our cohort. However, our study was limited by the small size and lack of follow-up data.

\section{Conclusion}

In our study, PD-L1 immunopositivity was found in $40 \%$ of patients and majority of them exhibited adenocarcinoma type of NSCLC. There was no correlation of PD-L1 expresion with age, gender, clinical stage, smoking status and tumor histology. There was a highly significant statistical association between adenocarcinoma subtype and PD-L1 expression, with the solid subtype correlating with positive PD-L1 expression. Our study also demonstrated that less tumor 
differentiation/poorly differentiated histology (G3) was significantly associated with PD-L1 positivity.

\section{Conflicts of Interest}

The authors declare no conflicts of interest regarding the publication of this paper.

\section{References}

[1] Inamura, K., Yokouchi, Y., Sakakibara, R., Kobayashi, M., Subat, S., Ninomiya, H., et al. (2016) Relationship of Tumor PD-L1 Expression with EGFR Wild-Type Status and Poor Prognosis in Lung Adenocarcinoma. Japanese Journal of Clinical Oncology, 46, 935-941. https://doi.org/10.1093/jico/hyw087

[2] Zhang, M., Li, G., Wang, Y., Wang, Y., Zhao, S., Haihong, P., et al. (2017) PD-L1 Expression in Lung Cancer and Its Correlation with Driver Mutations: A Meta-Analysis. Scientific Report, 7, 10255. https://doi.org/10.1038/s41598-017-10925-7

[3] Dela Cruz, C.S., Tanoue, L.T. and Matthay, R.A. (2011) Lung Cancer: Epidemiology, Etiology, and Prevention. Clinics in Chest Medicine, 32, 605-644. https://doi.org/10.1016/j.ccm.2011.09.001

[4] de Groot, P.M., Wu, C.C., Carter, B.W. and Munden, R.F. (2018) The Epidemiology of Lung Cancer. Translational Lung Cancer Research, 7, 220-233. https://doi.org/10.21037/tlcr.2018.05.06

[5] Sun, S., Schiller, J.H. and Gazdar, A.F. (2007) Lung Cancer in Never Smokers: A Different Disease. Nature Reviews Cancer, 7, 778-790. https://doi.org/10.1038/nrc2190

[6] Gabrielson, E. (2006) Worldwide Trends in Lung Cancer Pathology. Respirology, 11, 533-538. https://doi.org/10.1111/j.1440-1843.2006.00909.x

[7] Rudin, C.M., Avila-Tang, E., Harris, C.C., Herman, J.G., Hirsch, F.R., Pao, W., et al. (2009) Lung Cancer in Never Smokers: Molecular Profiles and Therapeutic Implications. Clinical Cancer Research, 15, 5646-5661. https://doi.org/10.1158/1078-0432.CCR-09-0377

[8] Sui, H., Ma, N., Wang, Y., Li, H., Liu, X., Su, Y., et al. (2018) Anti-PD-1/PD-L1 Therapy for Non-Small-Cell Lung Cancer: Toward Personalized Medicine and Combination Strategies. Journal of Immunology Research, 2018, Article ID: 6984948. https://doi.org/10.1155/2018/6984948

[9] Sharma, P. and Allison, J.P. (2015) Immune Checkpoint Targeting in Cancer Therapy: Towards Combination Strategies with Curative Potential. Cell, 161, 205-214. https://doi.org/10.1016/j.cell.2015.03.030

[10] Yang, J., Chen, J., Wei, J., Liu, X. and Cho, W.C. (2016) Immune Checkpoint Blockade as a Potential Therapeutic Target in Non-Small Cell Lung Cancer. Expert Opinion on Biological Therapy, 16, 1209-1223. https://doi.org/10.1080/14712598.2016.1214265

[11] Vallonthaiel, A.G., Malik, P.S., Singh, V., Kumar, V., Kumar, S., Sharma, M.C., et al. (2017) Clinicopathologic Correlation of Programmed Death Ligand-1 Expression in Non-Small Cell Lung Carcinomas: A Report from India. Annals of Diagnostic Pathology, 31, 56-61. https://doi.org/10.1016/j.anndiagpath.2017.07.001

[12] Teixidó, C., Vilariño, N., Reyes, R. and Reguart, N. (2018) PD-L1 Expression Testing in Non-Small Cell Lung Cancer. Therapeutic Advances in Medical Oncology, 
10, 1758835918763493. https://doi.org/10.1177/1758835918763493

[13] Sacher, A.G. and Gandhi, L. (2015) PD-1 and PD-L1 Inhibitors in Advanced Non-Small Cell Lung Cancer-Promising Agents and Evolving Questions. Oncology \& Hematology Review, 11, 36-42. https://doi.org/10.17925/OHR.2015.11.01.36

[14] Yu, H., Boyle, T.A., Zhou, C., Rimm, D.L. and Hirsch, F.R. (2016) PD-L1 Expression in Lung Cancer. Journal of Thoracic Oncology, 11, 964-975. https://doi.org/10.1016/j.jtho.2016.04.014

[15] Velcheti, V., Schalper, K.A., Carvajal, D.E., Anagnostou, V.K., Syrigos, K.N., Sznol, M., et al. (2014) Programmed Death Ligand-1 Expression in Non-Small Cell Lung Cancer. Laboratory Investigation, 94, 107-116.

https://doi.org/10.1038/labinvest.2013.130

[16] Yang, C.Y., Lin, M.W., Chang, Y.L., Wu, C.T. and Yang, P.C. (2014) Programmed Cell Death-Ligand 1 Expression in Surgically Resected Stage I Pulmonary Adenocarcinoma and Its Correlation with Driver Mutations and Clinical Outcomes. $E u^{-}$ ropean Journal of Cancer, 50, 1361-1369. https://doi.org/10.1016/j.ejca.2014.01.018

[17] Mu, C.Y., Huang, J.A., Chen, Y., Chen, C. and Zhang, X.G. (2011) High Expression of PD-L1 in Lung Cancer May Contribute to Poor Prognosis and Tumor Cells Immune Escape through Suppressing Tumor Infiltrating Dendritic Cells Maturation. Medical Oncology, 2, 682-688. https://doi.org/10.1007/s12032-010-9515-2

[18] Akbay, E.A., Koyama, S., Carretero, J., Altabef, A., Tchaicha, J.H., Christensen, C.L., et al. (2013) Activation of the PD-1 Pathway Contributes to Immune Escape in EGFR-Driven Lung Tumors. Cancer Discovery, 3, 1355-1363. https://doi.org/10.1158/2159-8290.CD-13-0310

[19] Calles, A., Liao, X., Sholl, L., et al. (2014) Differential Expression of LKB1, PD-L1, and PD-L2 in KRAS-Mutant Non-Small Cell Lung Cancer in Never-Smokers. ASCO Annual Meeting, Chicago, IL, June 2014. https://doi.org/10.1200/jco.2014.32.15_suppl.8032

[20] D’Incecco, A., Andreozzi, M., Ludovini, V., Rossi, E., Capodanno, A., Landi, L., et al. (2015) PD-1 and PD-L1 Expression in Molecularly Selected Non-Small-Cell Lung Cancer Patients. British Journal of Cancer, 112, 95-102. https://doi.org/10.1038/bjc.2014.555

[21] Pan, Y., Zheng, D., Li, Y., Cai, X., Zheng, Z., Jin, Y., et al. (2017) Unique Distribution of Programmed Death Ligand 1 (PD-L 1) Expression in East Asian Non-Small Cell Lung Cancer. The Journal of Thoracic Disease, 9, 2579-2586.

https://doi.org/10.21037/jtd.2017.08.61 